\title{
Reproductive value, sensitivity, and nonlinearity: Population-management heuristics derived from classical demography
}

\author{
Richard Karsten ${ }^{\mathrm{a}}$, Holger Teismann ${ }^{\mathrm{a}, \mathrm{b}, *}$, Angela Vogels ${ }^{\mathrm{a}}$ \\ a Acadia University, Wolfville, Canada \\ b Basque Center for Applied Mathematics, Bilbao, Spain
}

\section{A R T I C L E I N F O}

\section{Article history:}

Received 17 August 2012

Available online 18 January 2013

\section{Keywords:}

Age structure

Sharpe-Lotka-McKendrick equation

Density dependence

Sensitivity

Reproductive value

Population management

\begin{abstract}
A B S T R A C T
In classical demographic theory, reproductive value and stable age distribution are proportional to the sensitivities of the asymptotic population size to changes in mortality and maternity, respectively. In this note we point out that analogous relationships hold if the maternity function is allowed to depend on the population density. The relevant formulae can essentially be obtained by replacing the growth rate ("Lotka's r") with zero. These facts may be used to derive heuristics for population management (pest control).
\end{abstract}

(C) 2013 Elsevier Inc. All rights reserved.

\section{Introduction}

It has been discovered and pointed out multiple times ${ }^{1}$ that the reproductive value in the classical (linear) demographic theory of age-classified populations emerges as the sensitivity of the asymptotic growth rate ("Lotka's r") to changes in mortality.

The purpose of the present note is to demonstrate that the relationship between reproductive value and sensitivities has a nice generalization to nonlinear models; to point out that the interpretation of the reproductive value in terms of sensitivities is particularly simple and transparent; and to discuss these facts within the framework of population management (pest control).

Specifically, we describe an intuitive and beautiful correspondence between the classical linear theory of the reproductive value and its nonlinear generalization, which arises by considering a density-dependent maternity term. If such a term is included, the total population will eventually approach (or oscillate about) a

\footnotetext{
* Corresponding author at: Acadia University, Wolfville, Canada.

E-mail address: hteisman@acadiau.ca (H. Teismann).

1 A recent reference is Caswell (2010), which contains some history of the subject and further references. See also Banks et al. (2009) for rigorous derivations in the framework of PDE theory and Caswell $(2008,2009)$ for an in-depth treatment of nonlinear matrix models. In this note we use classical Lotka-Fisher-McKendrick theory (continuous in age and time) and adopt the notation of Caswell (2010).
}

non-zero stationary-state level $N$ and one can study the sensitivities of $N$ just as one studies the sensitivities of $r$. The correspondence principle to be presented says that the formulae for the nonlinear case are essentially (i.e. up to constant factors) identical to the known formulae for the linear case if $r$ is replaced with zero. This correspondence principle is intuitively appealing, as (a) both $r$ and $N$ describe the asymptotic states of the linear and nonlinear dynamics, respectively; (b) the population does not grow once it has reached $N$.

Our discussion will suggest that the reproductive value $v(x)$ may be best thought of in terms of asymptotic population size (denoted by $P$ below): it appears as the asymptotic size of a population that is subjected to harvesting age- $x$ individuals; it also appears as the asymptotic size of a population whose initial age distribution is modified by introducing age- $x$ individuals. We feel that these "operational" descriptions of the reproductive value offer valuable alternatives to the better-known definitions traditionally used in the literature. Furthermore, strictly speaking it is not the reproductive value that is proportional to the sensitivity of $r$, but what Ediev (2010) calls the "expected relative future demographic potential of the newborn" (see Eq. (5j) below).

The fact that the reproductive value is related to age-specific interventions (harvesting) suggests that it may provide simple heuristics for population management and control. We point out that this is not only true in the classical case, where the underlying demographic model is linear, but also in the case of a nonlinear model with a density-dependent maternity term. 
This paper is organized as follows. In Section 2 we provide the basic definitions used in this paper, including a list of the relevant demographic quantities. Section 3 contains the formulae for the sensitivities of $r, P$, and $N$ with respect to harvesting, mortality, and maternity in both the linear and nonlinear cases. Derivations of (some of) these identities are provided in Section 4. In Section 5 the results are illustrated by means of a textbook example from ecology: Nicholson's blowflies. The final two sections of the paper contain discussions of the results in the context of pest control applications and control theory (Section 6), as well as some generalizations (Section 7).

\section{Basic definitions}

We consider the nonlinear demographic model (continuous in both the time and age variables $t, a \in[0, \infty)$ ) for an age-classified population with density-dependent maternity (or fecundity)

$\rho_{t}+\rho_{a}=-\mu(a) \rho-h(a)$

$\rho(t, 0)=f(n(t)) \int_{0}^{\infty} m(a) \rho(t, a) d a$

$n(t)=\int_{0}^{\infty} v(a) \rho(t, a) d a$

Here $\rho=\rho(t, a)$ is the population density; the non-negative functions $m(a), \mu(a)$, and $h(a)$ are the age-specific fecundity, death, and harvesting rates, respectively. The function $f(n)$ represents the reduction in fecundity due to population size; it is assumed to be a positive and decreasing function satisfying $f(0)=1$. The non-negative function $v(a)$ specifies how the different age groups contribute to the nonlinear effect. For $v(a) \equiv 0$, the nonlinear term $f$ becomes one and (1a)-(1b) reduces to the classical (linear) Sharpe-Lotka-McKendrick equation (Webb, 1985).

We also define the total population and asymptotic population size by $p(t)=\int_{0}^{\infty} \pi(a) \rho(t, a) d a$ and $P=\lim _{t \rightarrow \infty} e^{-r t} p(t)$, respectively, where $\pi(a)$ is another non-negative function, which parametrizes the age-specific value - or burden, in the case of pest populations - of individuals of age $a$.

Both functions $\nu(a)$ and $\pi(a)$ incorporate the idea of "total population size", allowing for different age groups to be weighted differently. In Section 5 below, $v(a)$ and $\pi(a)$ are chosen to be simple step functions.

For the remainder of this section we assume that there is no harvesting; i.e. that $h(a) \equiv 0$. Then the stationary-state solution, $\rho(t, a) \equiv \rho_{0}(a)$, of $(1 \mathrm{a})$ is given by

$\rho_{0}(a)=C e^{-\int_{0}^{a} \mu(b) d b}=C \ell(a)$

(the constant $C$ is fixed by condition (4) below). Condition (1b) implies that the weighted equilibrium population level

$N_{0}=\int_{0}^{\infty} v(a) \rho_{0}(a) d a$

satisfies the equation

$f\left(N_{0}\right)=\frac{1}{R_{0}}$

where

$R_{0}=\int_{0}^{\infty} m(a) \ell(a) d a$

is the net reproduction rate. We assume $R_{0}>1$ so that the population is growing (at least until it reaches the equilibrium level $N_{0}$ ).

In view of (4) and (1b), the effect of the nonlinearity may be interpreted as switching the maternity function from $m(x)$ at low population densities to the "level of bare replacement" (Keyfitz, 1971) $m(x) / R_{0}$ at the equilibrium level $N_{0}$.
We conclude this section by listing the additional demographic quantities used in this paper (following the notation of Caswell (2010, 1.A)).

$\ell(x)=e^{-\int_{0}^{x} \mu(a) d a} \quad$ survivorship

$1=\int_{0}^{\infty} m(a) e^{-r a} \ell(a) d a \quad$ Euler-Lotka equation

$b=\left[\int_{0}^{\infty} e^{-r a} \ell(a) d a\right]^{-1} \quad$ birth rate ${ }^{2}$

$\stackrel{\circ}{b}=[b(x)]_{r=0}=\left[\int_{0}^{\infty} \ell(a) d a\right]^{-1}$

zero-growth birth rate ${ }^{2}=$ death rate $^{3}$

$c(x)=b e^{-r x} \ell(x)$ stable age distribution

$\check{c}(x)=[c(x)]_{r=0}=\stackrel{b}{\ell}(x)$ stationary-state age distribution

$v(x)=\frac{1}{e^{-r x} \ell(x)} \int_{x}^{\infty} e^{-r a} m(a) \ell(a) d a$ reproductive value

$\dot{v}(x)=[v(x)]_{r=0}=\frac{1}{\ell(x)} \int_{x}^{\infty} m(a) \ell(a) d a$

zero-growth reproductive value

$u(x)=c(x) v(x) / b=\int_{x}^{\infty} m(a) e^{-r a} \ell(a) d a$

demographic potential

$\stackrel{\circ}{u}(x)=[u(x)]_{r=0}=\int_{x}^{\infty} m(a) \ell(a) d a$

zero-growth demographic potential

$\bar{A}=\int_{0}^{\infty} a m(a) e^{-r a} \ell(a) d a$ mean age of reproduction.

Remarks. (a) Readers unfamiliar with mathematical demography may wish to consult Section 5 for explicit expressions of the demographic quantities in the simple case of an insect population with two life stages.

(b) It is interesting to note that the "zero-growth" (circled) quantities have interpretations in terms of "cohorts". If a cohort initially consists of $c_{0}$ individuals, its size after $t$ time units is $c_{0} \ell(t)=\stackrel{\circ}{c}(t) c_{0} / \stackrel{\circ}{b}$. The quantity $c_{0} / \dot{b}=c_{0} \stackrel{\circ}{e}_{0}=c_{0} \int_{0}^{\infty} \ell(t) d t$ represents the total accumulated time lived by all individuals of the cohort; hence $\stackrel{\circ}{e}_{0}=1 / b$ is the expected life time lived by an (average) individual, or the "expectation of life" (Keyfitz, 1971). The quantity $c_{0} \stackrel{u}{u}(t)$ represents the number of offspring produced by the cohort after time $t$, so $\stackrel{u}{u}(x)$ is the expected number of offspring produced by an (average) individual after age $x$. Similarly, $\stackrel{v}{v}(x)=$ $c_{0} \stackrel{\imath}{u}(x) /\left(c_{0} \ell(x)\right)$ represents expected number of offspring produced by an individual after age $x$, where the expectation is relative to the number of individuals that have lived to age $x$.

\section{Relationships and correspondence}

To list the results, we adopt the notation and definitions of Caswell (2010). If $\alpha$ is a demographic quantity (such as $r, P$ or

\footnotetext{
2 Using the Lotka-Euler equation (5c), the formulae for $b$ and $\dot{b}$ have the interpretation (total number of births)/(total size of population) for populations that have assumed the stable and stationary-state age distributions, respectively. This explains the term "birth rate" in (5d) and (5e)

3 The fact that the population's birth and death rates are equal reflects the fact that its growth rate is zero.
} 
$N$ ) and $\beta(a)$ a life-table function (such as $\mu(a)$ or $m(a)$ ), we set

$\beta(\theta, a)=\beta(a)+\theta \delta(a-x) \quad(x>0)$

and

$\frac{d \alpha}{d \beta(x)}=\left.\frac{d \alpha(\theta)}{d \theta}\right|_{\theta=0}$

where $\delta$ denotes the $\delta$-"function" and $\alpha(\theta)$ is the demographic quantity $\alpha$ associated with (1a)-(1c) if $\beta(a)$ is replaced with $\beta(\theta, a)$. In the case of harvesting, the perturbation is applied to the no-harvesting situation; i.e., we set

$h(\theta, a)=\theta \delta(a-x)$.

As in (3), we define

$N=\int_{0}^{\infty} v(a) \rho(a) d a \stackrel{*}{=} \lim _{t \rightarrow \infty} n(t)$,

where $\rho(a)$ is a stationary-state solution of (1a)-(1c) and the identity marked with an asterisk holds if the equilibrium $N$ is stable. Moreover, we write $N_{0}$ for $\left.N\right|_{\theta=0}$ and

$\pi_{0}=\int_{0}^{\infty} \pi(a) e^{-r a} \ell(a) d a, \quad$ and $\quad \stackrel{\circ}{\nu}_{0}=\int_{0}^{\infty} v(a) \ell(a) d a$.

With these notations we have the following identities, which we organize in a table to highlight the analogies between the linear and nonlinear cases. Derivations will be given in the next section.

\begin{tabular}{|c|l|l|l|}
\hline & $\begin{array}{l}\text { Sensitivity } \\
\text { to }\end{array}$ & Linear & Nonlinear \\
\hline I & Harvesting & $\frac{d P}{d h(x)}=-\frac{\pi_{0}}{r \bar{A}} v(x)$ & $\frac{d N}{d h(x)}=\frac{\stackrel{\circ}{0}_{0}}{R_{0}^{2} f^{\prime}\left(N_{0}\right) N_{0}} \stackrel{v}{ }(x)$ \\
\hline II & Mortality & $\frac{d r}{d \mu(x)}=-\frac{1}{\bar{A}} u(x)$ & $\frac{d N}{d \mu(x)}=\frac{1}{R_{0}^{2} f^{\prime}\left(N_{0}\right)} \stackrel{u}{u}(x)$ \\
\hline III & Maternity & $\frac{d r}{d m(x)}=\frac{1}{b \bar{A}} c(x)$ & $\frac{d N}{d m(x)}=-\frac{1}{R_{0}^{2} f^{\prime}\left(N_{0}\right) \grave{b}} \stackrel{c}{c}(x)$ \\
\hline
\end{tabular}

Remarks. (a) Formula I (linear) holds for any non-negative function $\pi(a)$, including $\pi(a)=v(a)$, in which case $p(t)=n(t)$.

(b) The table may appear asymmetrical in rows II and III of the first column, where one might expect to see $d P / d \mu(x)$ and $d P / d m(x)$. However, because of the exponential growth of the population, the changes in $r$ caused by variations of $\mu$ or $m$ will always dominate the asymptotic value of $p(t)$. So $d r / d \mu(x)$ and $d r / d m(x)$ are the relevant quantities with respect to the asymptotic size of the population. (Note, by contrast, that in scenario I the growth rate $r$ is not affected by the harvesting term.)

(c) The sensitivities of $N$ in the "nonlinear column" may be replaced with the sensitivities of $P$, if "formal" maternity functions are used instead of $m(x)$. Details are given in Section 7.1 below.

(d) Formulae II and III (linear) coincide with Caswell $(2010,2.1)-$ except for the use of $u(x)$ in II, which in Caswell (2010) is written as $c(x) v(x) / b$.

\section{Derivation}

We restrict ourselves to verifying the formulae in the first row. The linear versions of formulae II and III are proved in Caswell (2010); the nonlinear cases are readily verified by implicitly differentiating the identity $1 / f(N)=\int_{0}^{\infty} m(a) \ell(a) d a$ (cf. Eq. (4)). To study the asymptotic size of the population subject to harvesting, we consider Eq. (1a) with $h(\theta, a)$ given by (6).
Linear. By the Sharpe-Lotka Theorem (Webb, 1984), $\rho$ is asymptotically given by

$\rho(t, a)=\frac{Q}{b \bar{A}} e^{r t} c(a) \quad($ as $t \rightarrow \infty)$,

where

$$
\begin{aligned}
Q & =\int_{0}^{\infty} v(a) g(a) d a-\frac{1}{r} \int_{0}^{\infty} v(a) h(\theta, a) d a \\
& =\int_{0}^{\infty} v(a) g(a) d a-\frac{\theta}{r} v(x),
\end{aligned}
$$

and $g(a)=\rho(0, a)$ is the initial age distribution. Thus

$P=\lim _{t \rightarrow \infty} e^{-r t} p(t)=\frac{Q}{\bar{A}} \int_{0}^{\infty} \pi(a) e^{-r a} \ell(a) d a=\frac{Q}{\bar{A}} \pi_{0}$

and so

$\left.\frac{d P}{d \theta}\right|_{\theta=0}=-\frac{\pi_{0}}{r \bar{A}} v(x)$.

Remark. The proof shows that $P$ itself (not only its derivative) is actually proportional to a translation of $v(x)$.

Nonlinear. The stationary-state age distribution is now given by $^{4}$

$$
\begin{aligned}
\rho(a) & =\ell(a)\left[C-\int_{0}^{a} h(\theta, y) / \ell(y) d y\right] \\
& =\ell(a)[C-\theta H(a-x) / \ell(x)],
\end{aligned}
$$

which gives

$$
\begin{aligned}
N & =\int_{0}^{\infty} v(a) \rho(a) d a \\
& =C \int_{0}^{\infty} v(a) \ell(a) d a-\frac{\theta}{\ell(x)} \int_{x}^{\infty} v(a) \ell(a) d a \\
& =C \psi(0)-\theta \psi(x)
\end{aligned}
$$

with $\psi(x)=\frac{1}{\ell(x)} \int_{x}^{\infty} v(a) \ell(a) d a$. From (1b) and (10) we find that

$C=f(N)\left[C R_{0}-\theta \dot{v}(x)\right]$.

Combining (11) and (12) yields an implicit formula for $N$

$N+\theta \psi(x)=R_{0} f(N)[N+\theta \psi(x)]-\theta \psi(0) f(N) \stackrel{\circ}{v}(x)$.

Now it is a routine calculation (see Vogels (2011)) to show that applying $\left.\frac{d}{d \theta}\right|_{\theta=0}$ to both sides of (13) and using (4) and $\psi(0)=\stackrel{\circ}{\nu}_{0}$ results in Eq. I, Nonlinear.

\section{Example: Nicholson's blowflies}

A prominent example of a (st)age-structured population with density-dependent maternity (fecundity) is Nicholson's blowflies (Nicholson, 1954a,b), which has been successfully modelled (Perez et al., 1978; Gurney et al., 1980) by delay-differential equations of the form

$\frac{d}{d t} \tilde{n}(t)=m e^{-\mu a} e^{-v \tilde{n}(t-a)} \tilde{n}(t-a)-\mu \tilde{n}(t)$

( $m, \mu, a, v$ positive constants).

This equation can be derived from (1a)-(1c) by the "linear chain trick" (Cushing, 1998), if $\mu(x) \equiv \mu, m(x)=m H(x-a)$ and

\footnotetext{
${ }^{4} H(s)$ denotes the Heaviside function, which may formally be defined as $H(s)=$ $\int_{-\infty}^{s} \delta(t) d t$; i.e., $H(s)=0$ for $s<0$ and $H(s)=1$ for $s>1$.
} 


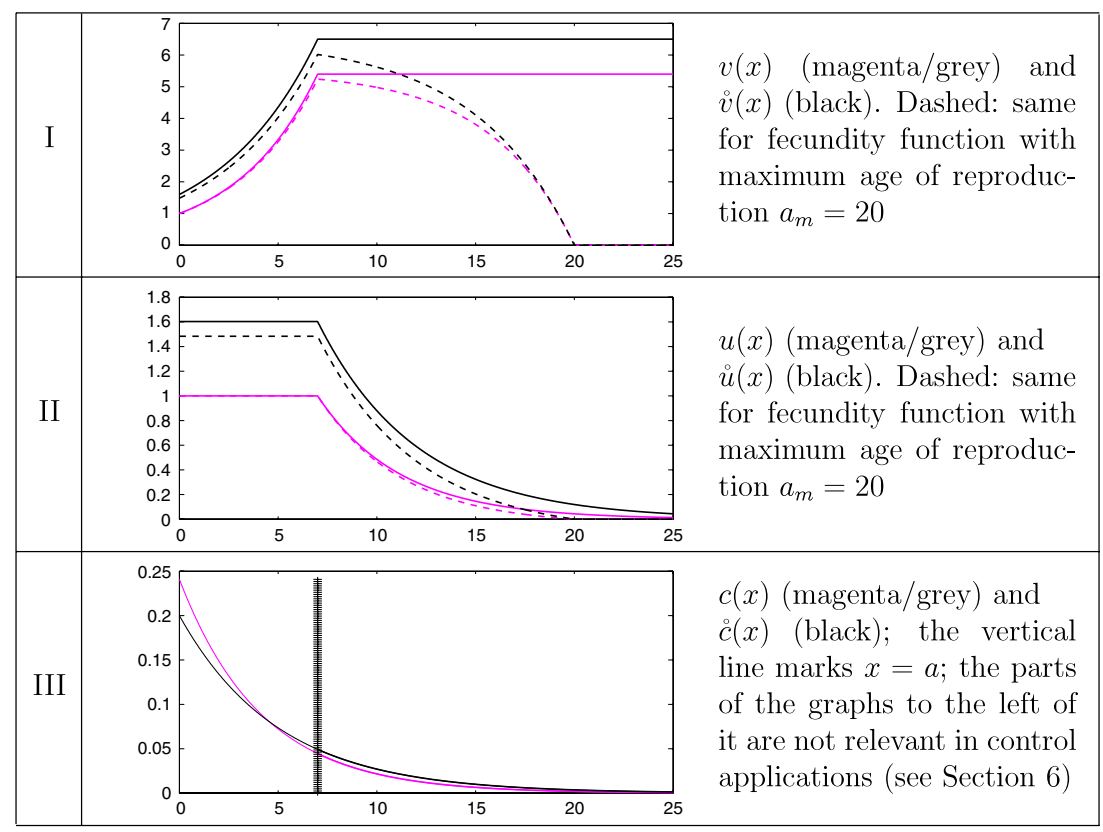

Fig. 1. Reproductive value, reproductive potential, and stable age distribution with their zero-growth counterparts.

$v(x)=v H(x-a), f(n)=e^{-n}$, and $\tilde{n}(t)=\int_{a}^{\infty} \rho(t, x) d x$ (here $n(t)=v \tilde{n}(t))$.

In this simple case, all demographic quantities can be computed explicitly:

$$
\begin{aligned}
& R_{0}=\frac{m e^{-\mu a}}{\mu} \\
& \ell(x)=e^{-\mu x} \\
& r+\mu=m e^{-(r+\mu) a} \\
& b=r+\mu \\
& \stackrel{\circ}{b}=\mu \\
& c(x)=(r+\mu) e^{-(r+\mu) x} \\
& \stackrel{\circ}{c}(x)=\mu e^{-\mu x} \\
& v(x)=\frac{m}{r+\mu}\left[H(a-x) e^{(r+\mu)(x-a)}+H(x-a)\right] \\
& \stackrel{(14 c)}{=} H(a-x) e^{(r+\mu) x}+H(x-a) e^{(r+\mu) a} \\
& \dot{v}(x)=\frac{m}{\mu}\left[H(a-x) e^{\mu(x-a)}+H(x-a)\right] \\
& u(x)=\frac{m}{r+\mu}\left[H(a-x) e^{-(r+\mu) a}+H(x-a) e^{-(r+\mu) x}\right] \\
& \stackrel{(14 \mathrm{c})}{=} H(a-x)+H(x-a) e^{-(r+\mu)(x-a)} \\
& \stackrel{\circ}{u}(x)=\frac{m}{\mu}\left[H(a-x) e^{-\mu a}+H(x-a) e^{-\mu x}\right] \\
& \bar{A}=a+\frac{1}{r+\mu} .
\end{aligned}
$$

Moreover,

$N_{0}=\ln \left(R_{0}\right)=\ln \left(\frac{m}{\mu}\right)-\mu a, \quad \stackrel{\circ}{\nu}_{0}=\frac{v e^{-\mu a}}{\mu}, \quad$ and $\frac{1}{R_{0}^{2} f^{\prime}\left(N_{0}\right)}=\frac{-1}{R_{0}}$.

Plots of the function $v(x), \stackrel{\circ}{v}(x), u(x), \stackrel{\circ}{u}(x), c(x)$, and $\stackrel{\circ}{c}(x)$ for realistic parameter values are provided in Fig. 1.
Remarks. (a) The fact that $v(x)$ and $\dot{v}(x)$ are constant for $x \geq a$ is a result of the unrealistic assumption that $m(x)$ is constant for $x \geq a$. For more realistic fecundity functions $m(x)$, which decline for large $x, v(x)$ and $\dot{v}(x)$ also decrease. The broken lines in the first two plots of Fig. 1 show the graphs of $v(x)$ and $\dot{v}(x)$ (resp. $u(x)$ and $\mathfrak{u}(x)$ ) if a maximum age of reproduction $a_{m}>a$ is introduced; i.e., if $m(x)=m H(x-a)$ is replaced with $m(x)=m\left[H(x-a)-H\left(a_{m}-x\right)\right]$. (The functions $c(x)$ and $\stackrel{\circ}{c}(x)$ are not affected by the introduction of $a_{m}$.)

(b) Note that $u(x)$ and $\stackrel{\circ}{u}(x)$ always satisfy $u(x) \equiv 1$ and $\stackrel{u}{u}(x) \equiv R_{0}$ for $x$ below the minimum age of reproduction; i.e., for $x \leq a$.

\section{Discussion}

This research grew out of a interdisciplinary research project on mite infestations in apple orchards. We are therefore thinking of $\rho$ as representing the population density of a fast-growing, stagestructured species, such as mites or insects, and we are interpreting the sensitivities with respect to "harvesting", "mortality", and "maternity" as the application of (biological or chemical) control measures. If we assume that we are able to design control strategies that are specific to one age group $x$ (i.e. that only attack age- $x$ individuals), it is natural to ask which age group $x$ should be targeted to have an optimal control effect. The results of Section 3 can then be interpreted as follows.

I. Harvesting. The reduction in the asymptotic population size $P$ as a result of harvesting individuals of age $x$ is proportional to the reproductive value $v(x)$.

II. Mortality. The reduction in $P$ as a result of introducing a predator species or applying a pesticide that attacks individuals of age $x$ is proportional to the reproductive potential $u(x)$.

III. Maternity. The reduction in $P$ as a result of diminishing the fecundity of age- $x$ individuals is proportional to the stable age distribution $c(x)$. Here $x$ should be chosen larger than the minimum age of reproduction, since, biologically, it makes little sense to consider the reduction of the fecundity of nonreproductive individuals. 
According to the correspondence principle presented in the previous sections, these statements remain valid in the nonlinear case, if $P$ is replaced with the equilibrium population level $N$ and the quantities $v(x), u(x)$ and $c(x)$ are replaced with their "zero-growth" counterparts $\stackrel{v}{v}(x), \stackrel{u}{u}(x)$, and $\stackrel{c}{c}(x)$, respectively ${ }^{5}$. It is remarkable that the optimal age class to be targeted (to achieve the largest reduction in population) can be expressed in terms of quantities of classical ("linear") demographic theory.

In particular, the largest possible reduction is achieved if the age group is targeted that corresponds to the maximum of the function in question. For II this is any pre-reproductive age group; for III this is the first reproductive age group, since considering prereproductive age groups does not make sense biologically; and even for scenario I it is often the case that the functions $v(x)$ and $\stackrel{v}{v}(x)$ have their maxima close to the minimum age of reproduction. This means that, for all three control methods, targeting the first reproductive age group will "simultaneously" reduce linear growth $(r)$ and equilibrium level $(N)$ in an optimal fashion.

In Teismann et al. (2009) we demonstrated that for a twostage population with nonlinear fecundity, which oscillates about a positive equilibrium, the population mean might respond in an unexpected way to control measures: increasing mortality may actually increase the mean rather than reduce it, as one would naively expect. (This phenomenon has recently been called "hydra effect" (Abrams, 2009).) It would therefore be interesting to examine the sensitivities of the population mean with respect to harvesting, mortality, and maternity. This, as well as studying the effect of relaxing other basic assumptions of the classical Lotka-Fisher theory of the reproductive value (such as timeindependent life-table data), is the subject of ongoing research by the authors.

A "pedagogical" comment concerns the concept of reproductive value, which often proves a difficult one. The concept is traditionally introduced by either referring to Fisher's (Fisher, 1930) original "loan-interest" explanation ${ }^{6}$, or by giving a somewhat formal algebraic definition as the left eigenvector of the projection matrix. We feel that statement I above (sensitivity of $P$ with respect to harvesting) provides a simple "operational" definition of the reproductive value, which can complement the better-known definitions traditionally used. (Another such "operational" definition is afforded by the sensitivity of $P$ with respect to the initial age distribution, which follows from (9a)-(9b). $)^{7}$ The approach taken in this paper emphasizes these operational explanations of the reproductive value. Our approach may be called "bottom-up" in that we start with classical demographic theory and study the extent to which it gives useful information for control applications. A "topdown" approach has been taken by other researchers who first apply full-fledged mathematical control theory and then, after the fact, interpret the outcomes in terms of known demographic quantities such as the reproductive value (see e.g. Hadeler and Müller (2007), Wrzaczek et al. (2010)).

In keeping with our approach, we consider a nonlinear model that not only reflects the biology of our real-world application, but may also be interpreted in terms of classical demography: as explained above, the nonlinear term may be viewed as a nonlinear (negative-feedback) version of the Keyfitz-scenario of switching the reproductive rate to the "level of bare replacement". Within this interpretation the " $r=0$ rule" becomes particularly

\footnotetext{
5 There are also explicit formulae for the sensitivities of $P$ in the nonlinear case. These are listed in Section 7.1.

6 Caswell (2001) comments on this supposedly "easy" derivation: "With all due respect to Fisher, I have yet to meet anyone who finds this equation "easily seen"'.

7 For a discussions of the various interpretations of the reproductive value, see also Vogels (2011) and the literature cited therein.
}

intuitive. At the same time, it is an interesting and surprising observation that the sensitivities of the equilibrium level can still be expressed in terms of the reproductive value. This implies that using reproductive-value-based heuristics in control applications may be a sensible strategy, even if the underlying demographic model is nonlinear.

\section{Epilogue: some generalizations}

The point of the present paper is distinctly not to present results of the greatest possible generality. Rather, it is to highlight intriguing parallels between linear and nonlinear demographic theory in the special situation of a uniform (i.e. age-independent) density-dependence in the maternity function.

However, one might wonder to what extent the methods and results presented depend on these particular assumptions. The purpose of this additional section (which is partially motivated by the referees' comments) is to consider a few obvious generalizations and to convince the reader that the simple techniques of this paper may be applied to various scenarios. Again, we are not striving for "optimal" results, but leave it to the intrepid reader to work out her/his favorite case.

\subsection{Sensitivities of $P$ in the nonlinear case}

In Section 3 we implicitly made the choice $\pi(x) \equiv v(x)$ (i.e. $P=$ $N)$. In the general case, we obtain formulae for the sensitivities of $P$ whose formal structure (including factors) coincides with the formulae for the sensitivities of $N$, if the actual biological maternity function $m(x)$ is replaced with a new - formal - one. The formulae for these fictitious "maternity" functions are listed below (where $\left.\stackrel{\circ}{\pi}_{0}=\int_{0}^{\infty} \pi(a) \ell(a) d a\right)$. Note that these expressions reduce to $m(x)$ if $\pi(x) \equiv v(x)$, as expected.

\begin{tabular}{|c|c|c|}
\hline & $\begin{array}{l}\text { Sensitivity } \\
\text { to }\end{array}$ & $\begin{array}{l}\text { Same as in the "Nonlinear" column if } \\
m(x) \text { is replaced with ... }\end{array}$ \\
\hline I & Harvesting & $\frac{\stackrel{\circ}{\pi}_{0}}{\stackrel{\circ}{\nu}_{0}} m(x)+\frac{R_{0}^{2} f^{\prime}\left(N_{0}\right) N_{0}}{\dot{\circ}_{0}} \cdot \frac{\stackrel{\circ}{\pi}_{0} v(x)-\stackrel{\circ}{\nu}_{0} \pi(x)}{\stackrel{\circ}{\nu}_{0}}$ \\
\hline II & Mortality & $\frac{\stackrel{\circ}{\pi}_{0}}{\stackrel{\circ}{\nu}_{0}} m(x)+\frac{R_{0}^{2} f^{\prime}\left(N_{0}\right) N_{0}}{\stackrel{\circ}{\nu}_{0}} \cdot \frac{\stackrel{\circ}{\pi}_{0} v(x)-\stackrel{\circ}{\nu}_{0} \pi(x)}{\stackrel{\circ}{\nu}_{0}}$ \\
\hline III & Maternity & $\frac{\stackrel{\circ}{\pi}_{0}}{\stackrel{\circ}{0}_{0}} m(x)$ \\
\hline
\end{tabular}

\subsection{Density-dependent mortality}

Scenarios that include density-dependent mortality can also be treated with similar methods. This can be accomplished by replacing the mortality function $\mu(x)$ in (1a) with a term of the form

$$
\tilde{\mu}(t, x)=\mu(x)+g(k(t)) \mu_{1}(x),
$$

where $g(k) \geq 0$ and $k(t)$ is defined by means of (yet) another weight function $\kappa(x) \geq 0$; i.e., $k(t):=\int_{0}^{\infty} \kappa(a) \rho(t, a) d a$. For simplicity, we restrict ourselves to the case $\kappa \equiv v$. We use the tilde notation to indicate the substitution

$\mu(x) \rightarrow \tilde{\mu}(x):=\mu(x)+g(\tilde{N}) \mu_{1}(x)$.

Normally, zero-subscripts are used to indicate evaluation at $\theta=$ 0 ; however, we drop the subscripts for the reproductive value, 
reproductive potential, and stable age distribution. Specifically,

$\tilde{\ell}(x)=e^{-\int_{0}^{x}\left[\mu(a)+g(\tilde{N}) \mu_{1}(a)\right] d a}$

$\tilde{N}=\tilde{C} \int_{0}^{\infty} v(a) \tilde{\ell}(a) d a$

$\tilde{R}=\int_{0}^{\infty} m(a) \tilde{\ell}(a)$

$\tilde{\tilde{v}}(x)=\frac{1}{\tilde{\ell}_{0}(x)} \int_{x}^{\infty} m(a) \tilde{\ell}_{0}(a) d a$

$\dot{\tilde{u}}(x)=\int_{x}^{\infty} m(a) \tilde{\ell}_{0}(a) d a$

$\tilde{b}(x)=\left[\int_{0}^{\infty} \tilde{\ell}_{0}(a) d a\right]^{-1}$

$\dot{\tilde{c}}(x)=\stackrel{\tilde{b}}{\tilde{\ell}_{0}}(a) d a$.

Moreover, let

$\tilde{H}_{0}:=f^{\prime}\left(\tilde{N}_{0}\right) \tilde{R}_{0}^{2}-g^{\prime}\left(\tilde{N}_{0}\right) \int_{0}^{\infty} m(a)\left(\int_{0}^{a} \mu_{1}(b) d b\right) \tilde{\ell}_{0}(a) d a$.

Then

\begin{tabular}{|c|c|c|c|}
\hline & Sensitivity to & Linear mortality & Nonlinear mortality \\
\hline I & Harvesting & $\frac{d N}{d h(x)}=\frac{\dot{\nu}_{0}}{R_{0}^{2} f^{\prime}\left(N_{0}\right) N_{0}} \stackrel{v}{ }(x)$ & $\frac{d \tilde{N}}{d h(x)}=\frac{\dot{\tilde{v}}_{0}}{\tilde{H}_{0} \tilde{N}_{0}} \dot{\tilde{v}}(x)$ \\
\hline II & Mortality & 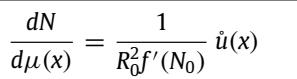 & $\frac{d \tilde{N}}{d \tilde{\mu}(x)}=\frac{1}{\tilde{H}_{0}} \stackrel{\tilde{u}}{(x)}$ \\
\hline III & Maternity & $\frac{d N}{d m(x)}=-\frac{1}{R_{0}^{2} f^{\prime}\left(N_{0}\right) \dot{b}} \stackrel{\circ}{c}(x)$ & $\frac{d \tilde{N}}{d m(x)}=-\frac{1}{\tilde{H}_{0} \tilde{\tilde{c}}} \dot{\tilde{c}}(x)$ \\
\hline
\end{tabular}

The $x$-dependences of the sensitivities can therefore be obtained by replacing $\mu(x)$ with $\tilde{\mu}_{0}(x):=\mu(x)+g\left(\tilde{N}_{0}\right) \mu_{1}(x)$. Note, however, that the quantities $\dot{\tilde{v}}(x), \dot{\tilde{u}}(x)$, and $\dot{\tilde{c}}(x)$ now depend on the equilibrium level $\tilde{N}_{0}$. As a result, the $x$-dependences are not simply function(al)s of the life table data $\mu$ and $m$, but also depend on the nonlinearity $g$.

\subsection{Non-uniform (i.e. age-dependent) nonlinearity}

Individuals of different ages may not only contribute differently to the nonlinear reduction in fecundity (as parametrized by $v(x)$ ), but their own fertility may also be affected differently. To discuss this we make the substitution

$f(n) \rightarrow \tilde{f}(n, a)$

and again adopt the tilde notation. Defining

$\dot{\tilde{v}}(x)=\frac{1}{\ell(x)} \int_{x}^{\infty} \tilde{f}\left(\tilde{N}_{0}, a\right) m(a) \ell(a) d a$

$\dot{\tilde{u}}(x)=\int_{x}^{\infty} \tilde{f}\left(\tilde{N}_{0}, a\right) m(a) \ell(a) d a$

$\dot{\tilde{c}}(x)=\tilde{f}\left(\tilde{N}_{0}, x\right) c(x)$

$\tilde{R}_{1}=\int_{0}^{\infty} \tilde{f}_{n}\left(\tilde{N}_{0}, a\right) m(a) \ell(a) d a$

(where $\tilde{f}_{n}$ denotes the partial derivative of $\tilde{f}$ with respect to the first variable), we obtain

\begin{tabular}{|c|c|c|c|}
\hline & Sensitivity to & Uniform nonlinearity & $\begin{array}{l}\text { Age-dependent } \\
\text { nonlinearity }\end{array}$ \\
\hline I & Harvesting & $\frac{d N}{d h(x)}=\frac{\dot{\nu}_{0}}{R_{0}^{2} f^{\prime}\left(N_{0}\right) N_{0}} \dot{v}(x)$ & $\frac{d \tilde{N}}{d h(x)}=\frac{\dot{\nu}_{0}}{\tilde{R}_{1} \tilde{N}_{0}} \tilde{v}(x)$ \\
\hline II & Mortality & 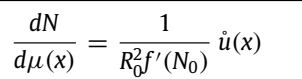 & $\frac{d \tilde{N}}{d \mu(x)}=\frac{1}{\tilde{R}_{1}} \check{\tilde{u}}^{\prime}(x)$ \\
\hline III & Maternity & $\frac{d N}{d m(x)}=-\frac{1}{R_{0}^{2} f^{\prime}\left(N_{0}\right) \dot{b}} \grave{c}(x)$ & 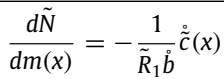 \\
\hline
\end{tabular}

As in the previous section, we note that the quantities $\stackrel{\tilde{v}}{(x),}, \stackrel{\tilde{u}}{(x)}$, and $\stackrel{\tilde{c}}{(}(x)$ depend on the equilibrium level $\tilde{N}_{0}$.

\section{Acknowledgments}

This research was supported by a Discovery Grant of the Natural Sciences and Engineering Research Council of Canada (NSERC); AV also acknowledges support by NSERC through a graduate scholarship. HT would like to thank the Basque Center for Applied Mathematics (BCAM) for its hospitality and financial support.

\section{References}

Abrams, P., 2009. When does greater mortality increase population size? The long history and diverse mechanisms underlying the hydra effect. Ecol. Lett. 12, 462-474.

Banks, H., Ernstberger, S., Hu, S., 2009. Sensitivity equations for a size-structured population model. Quart. Appl. Math. 67, 627-660.

Caswell, H., 2010. Reproductive value, the stable stage distribution, and the sensitivity of the population growth rate to changes in vital rates. Demogr. Res. 23, 531-548.

Caswell, H., 2008. Perturbation analysis of nonlinear matrix population models. Demogr. Res. 18, 59-116.

Caswell, H., 2009. Sensitivity and elasticity of density-dependent population models. J. Difference Equ. Appl. 15, 349-369.

Caswell, H., 2001. Matrix Population Models: Construction, Analysis, and Interpretation. Sinauer Associates.

Cushing, J., 1998. An Introduction to Structured Population Dynamics, Vol. 71. Society for Industrial Mathematics.

Ediev, D., 2010. On the reproductive value and the spectrum of a population projection matrix with implications for dynamic population models. Theor. Popul. Biol. 78, 67-70.

Fisher, R., 1930. The Genetical Theory of Natural Selection. Clarendon Press.

Gurney, M., Blythe, S., Nisbet, R., 1980. Nicholson's blowflies revisited. Nature 287, 17-21.

Hadeler, K., Müller, J., 2007. Optimal harvesting and optimal vaccination. Math. Biosci. 206, 249-272.

Keyfitz, N., 1971. On the momentum of population growth. Demography 8, 71-80.

Nicholson, A., 1954a. Compensatory reactions of populations to stress, and their evolutionary significance. Aust. J. Zool. 2, 1-8.

Nicholson, A., 1954b. An outline of the dynamics of animal populations. Aust. J. Zool. 2, 9-65.

Perez, J., Malta, C., Coutinho, F., 1978. Qualitative analysis of oscillations in isolated populations of flies. J. Theoret. Biol. 71, 505-514.

Teismann, H., Karsten, R., Hammond, R., Hardman, J., Franklin, J., 2009. On the possibility of counter-productive intervention: the population mean for blowflies models can be an increasing function of the death rate. J. Biol. Systems 17, 739-757.

Vogels, A., 2011. The Fundamentals and Theoretical Concepts of Modeling Age Structured Populations. Master's thesis. Acadia University, Wolfville, N.S., Canada.

Webb, G., 1985. Theory of Nonlinear Age-Dependent Population Dynamics, Vol. 89. CRC.

Webb, G., 1986. A semigroup proof of the Sharpe-Lotka theorem. In: Infinitedimensional Systems. In: Lecture Notes in Mathematics, vol. 1076. Springer.

Wrzaczek, S., Kuhn, M., Prskawetz, A., Feichtinger, G., 2010. The reproductive value in distributed optimal control models. Theor. Popul. Biol. 77, 164-170. 